Pacific Journal of Mathematic 


\title{
HOMOMORPHISMS OF SEMI-SIMPLE ALGEBRAS
}

\author{
JAMES D. STEIN, JR.
}

\begin{abstract}
Let $\nu: \mathfrak{U} \rightarrow \mathfrak{B}$ be a Banach algebra homomorphism of a semi-simple Banach algebra $\mathfrak{H}$. The purpose of this paper is to investigate certain topological properties of $\nu$ under various assumptions about $\mathfrak{U}$.
\end{abstract}

Given a Banach algebra homomorphism $\nu: \mathfrak{U} \rightarrow \mathfrak{B}$, let $S(\nu, \mathfrak{B})$ be the set of all $b \in \mathfrak{B}$ such that there is a sequence $\left\{x_{n} \in \mathfrak{U} \mid n=1,2, \cdots\right\}$ with $\lim _{n \rightarrow \infty} x_{n}=0, \lim _{n \rightarrow \infty} \nu\left(x_{n}\right)=b$; and let $S(\nu, \mathfrak{u})$ be the set of all $x \in \mathfrak{U}$ such that there is a sequence $\left\{x_{n} \in \mathfrak{U} \mid n=1,2, \cdots\right\}$ with $\lim _{n \rightarrow \infty} x_{n}=0, \lim _{n \rightarrow \infty} \nu\left(x_{n}\right)=\nu(x)$. Each of these sets is a two-sided closed ideal, in $\mathfrak{U}$ or in the closure of $\nu(\mathfrak{u})$, and the closed graph theorem shows that $\nu$ is continuous if and only if $S(\nu, \mathfrak{B})=(0)$.

This paper is divided into two sections. In the first it is shown that, if $\mathfrak{U}$ is a $\mathrm{B}^{*}$-algebra, then $S(\nu, \mathfrak{u})$ is the closure of the kernel of $\nu$, thus extending a result of Cleveland ([2], p. 1103), and that, if $\mathfrak{U}$ is a commutative regular semi-simple algebra and $\nu$ is an isomorphism, then $S(\nu, \mathfrak{U})=(0)$. The second section is devoted to an analysis of the Badé-Curtis [1] decomposition of homomorphisms of $C(X)$, the algebra of all continuous complex-valued functions on a compact Hausdorff space $X$.

I. Homomorphisms of $B^{*}$-algebras, Let $\nu: \mathfrak{U} \rightarrow \mathfrak{B}$ be a Banach algebra homomorphism of a $B^{*}$-algebra $\mathfrak{U}$, and let $\mathfrak{B}$ be the closure of $\nu(\mathfrak{U})$ (this latter condition will remain in force throughout the paper). Let $K$ denote the kernel of $\nu$. We recall that a commutative $B^{*}$-algebra is either the algebra of all continuous complex-valued functions with supremum norm on some compact Hausdorff space, or those which vanish at infinity on a locally compact Hausdorff space. We let $C(X)$ denote the former, and $\mathrm{C}_{0}(X)$ the latter.

The first lemma is an easy extension of a well-known result for compact Hausdorff spaces ([3], p. 93), and is stated without proof.

Lemma I.1. Let $X$ be a locally compact Hausdorff space, $I$ a closed ideal in $C_{0}(X)$. Then there is a closed set $X_{I} \subseteq X$ such that $I=\left\{f \in C_{0}(X) \mid f\left(X_{I}\right)=0\right\}$.

The following lemma enables us to locate useful elements in a closed ideal in $C_{0}(X)$, and is a consequence of Theorem 2.7.23 of [4].

Lemma I.2. Let $X$ be locally compact Hausdorff, $F$ a finite subset of $X$. Let $T(F)$ denote the set of all functions $f$ in $C_{0}(X)$ 
which vanish on some open neighborhood $N_{f}$ of $F$, the neighborhood depending on $f$, and let $M(F)=\left\{f \in C_{0}(X) \mid f(F)=0\right\}$. Let $A$ be an ideal in $C_{0}(X)$ such that $\bar{A}=M(F)$. Let $g \in T(F)$, and assume that $g$ vanishes outside a compact set. Then $g \in A$.

If $x \in K$, let $x_{n}=(1 / n) x$ for $n=1,2, \cdots$. Clearly $\lim _{n \rightarrow \infty} x_{n}=0$ and $\lim _{n \rightarrow \infty} \nu\left(x_{n}\right)=0=\nu(x)$, so $x \in S(\nu, \mathfrak{u})$. Since $S(\nu, \mathfrak{u})$ is a closed ideal, we therefore have $\bar{K} \subseteq S(\nu, \mathfrak{u})$.

Theorem I.1. $S(\nu, \mathfrak{u})=\bar{K}$.

Proof. Let $S=S(\nu, \mathfrak{u})$, and assume $\bar{K} \neq S$. By [4], Theorem 4.9.2, $S$ is a *-ideal, and is therefore the linear span of its self-adjoint elements. Since $S \neq \bar{K}$, we can therefore find a self-adjoint element $y$ in $S \sim \bar{K}$. Let $\mathfrak{H}_{0}=C_{0}(X)$ be the Banach algebra generated by $y$. Let $\nu_{0}=\nu \mid \mathfrak{U}_{0}$, and let $K_{0}=K \cap \mathfrak{u}_{0} . \quad \bar{K}_{0}$ is a closed ideal in $\mathfrak{u}_{0}$, and so there is a closed set $F$ such that $\bar{K}_{0}=\{f \in \mathfrak{U} \mid f(F)=0\}$. We now endeavor to show that $F=\varnothing$; this will show that $\mathfrak{U}_{0} \subseteq \bar{K}$, and consequently that $\bar{K}=S$.

We first show that $F$ is finite. If there is an infinite sequence $\left\{x_{n} \mid n=1,2, \cdots\right\}$ contained in $F$, we can choose sequences

$$
\left\{V_{n} \mid n=1,2, \cdots\right\} \text { and }\left\{U_{n} \mid n=1,2, \cdots\right\}
$$

of open sets such that $x_{n} \in U_{n} \subseteq \bar{U}_{n} \subseteq V_{n}$ and $\mathrm{m} \neq n \Rightarrow V_{m} \cap V_{n}=\varnothing$. By Urysohn's Lemma, choose functions $f_{n} \in C_{0}(X)$ such that $f_{n}\left(\bar{U}_{n}\right)=$ $1, f_{n}\left(V_{n}^{\prime}\right)=0$, and $0 \leqq f_{n} \leq 1$. Let $g_{n}=f_{n}^{1 / 3}$. Since $f_{n}(F) \neq 0$, clearly $\nu_{0}\left(f_{n}\right) \neq 0$. But since $m \neq n \Rightarrow g_{m} g_{n}=0$, by [2], Theorem 4.9, there is an integer $N$ such that $n \geqq N \Rightarrow \nu_{0}\left(f_{n}\right)=\nu_{0}\left(g_{n}^{3}\right)=0$. So $F$ must be finite.

Now assume that $F \neq \varnothing$. Since $X$ is locally compact, there is an open set $E$ such that $F \subseteq E$ and $\bar{E}$ is compact. Choose open sets $U$ and $V$ such that $F \subseteq U \subseteq \bar{U} \subseteq V \leqq \bar{V} \leqq E$. Define $p \in C_{0}(X)$ by $p(\bar{U})=1, p\left(V^{\prime}\right)=0,0 \leqq p \leqq 1$. Since $p(F) \neq 0, p \notin \bar{K}_{0}$, and hence $\nu(p) \neq 0$. We note that $\left(p^{2}-p\right)\left(U \cup V^{\prime}\right)=0$, so $p^{2}-p$ vanishes on a neighborhood of $F$ and outside the compact set $\bar{E}$. So, by Lemma I.2, we see that $p^{2}-p \in K_{0}$, and so $\nu\left(p^{2}-p\right)=0 \Rightarrow \nu(p)^{2}=\nu(p)$. We have thus found an element $p \in S$ such that $q=\nu(p)$ is a nonzero idempotent in $S(\nu, \mathfrak{B})$, since it is clear that $\nu(S) \subseteq S(\nu, \mathfrak{B})$.

Since $p \in S$, there is a sequence $\left\{x_{n} \in \mathfrak{U} \mid n=1,2, \cdots\right\}$ such that $\lim _{n \rightarrow \infty} x_{n}=0, \lim _{n \rightarrow \infty} \nu\left(x_{n}\right)=q=\nu(p)$. Since $\lim _{n \rightarrow \infty} x_{n}=0$, the spectrum of $x_{n}$, and consequently the spectrum of $\nu\left(x_{n}\right)$, eventually lies in a small neighborhood of 0 . Since $q$ is a nonzero idempotent, the spectrum of $q$ is either $\{0,1\}$ or $\{1\}$ and so, by a result of Newburgh quoted in [4], p. 37, the spectrum of $\nu\left(x_{n}\right)$ eventually has points arbitrarily close to 1 . This contradiction establishes the theorem. 
Now let $\nu: \mathfrak{U} \rightarrow \mathfrak{B}$ be an isomorphism of a commutative regular semisimple algebra $\mathfrak{u}$. We show that $S(\nu, \mathfrak{u})=(0)$.

THEOREM I.2. $S(\nu, \mathfrak{u})=(0)$.

Proof. Assume there is an $s \in S(\nu, \mathfrak{u})$ with $s_{\mathfrak{A}}^{\top} \neq 0$. Then there is a sequence $\left\{x_{n} \in \mathfrak{U} \mid n=1,2, \cdots\right\}$ such that

$$
\lim _{n \rightarrow \infty} x_{n}=0, \lim _{n \rightarrow \infty} \nu\left(x_{n}\right)=\nu(s) .
$$

Let $F$ denote the Badé-Curtis [1] singularity set of $\nu$, and let $f$ be a function in $\mathfrak{u}$ which is zero on a neighborhood of $F$. If we let $\mathfrak{H}_{0}$ denote the algebra of all functions in $\mathfrak{U}$ vanishing on that neighborhood, then by [1], Theorem $3.9, \nu$ is continuous on $\mathfrak{U}_{0}$, and so

$$
\lim _{n \rightarrow \infty} x_{n} f=0 \Rightarrow \lim _{n \rightarrow \infty} \nu\left(x_{n} f\right)=0 .
$$

But $\nu(s f)=\lim _{n \rightarrow \infty} \nu\left(x_{n} f\right)=0$, and since $\nu$ is an isomorphism, $s f=0$. Consequently the support of $s$ consists of isolated points. Select one such isolated point $p$, and multiply $s$ by a function $g$ which is $1 / s(p)$ on $p$ and zero elsewhere; the product $s g$ is an idempotent and is in $S(\nu, \mathfrak{U})$ but is nonzero, a contradiction to [2], p. 1102, and the fact that $\nu$ is an isomorphism.

Since there exist discontinuous isomorphisms of commutative regular semi-simple algebras ([1], pp. 597-598), we see that having $\mathrm{S}(v, \mathfrak{u})=(0)$ for an isomorphism is not enough to insure continuity of that isomorphism.

II. Homomorphisms of $C(X)$. Throughout this section we shall be concerned with a Banach algebra homomorphism $\nu: C(X) \rightarrow \mathfrak{B}, X$ a compact Hausdorff space. Using the Badé-Curtis [1] decomposition of $\nu$, it is possible to obtain further information about $\nu$. We write $\nu=\mu+\lambda$, where $\mu$ is the continuous, and $\lambda$ the singular, part of $\nu$. Let $R$ denote the Jacobson radical of $\mathfrak{B}=\overline{\nu(C(X))}$. By construction $\nu$ and $\mu$ agree on a dense subalgebra of $C(X)$.

In general, if $\varphi: \mathfrak{U} \rightarrow \mathfrak{B}$ is a Banach algebra homomorphism such that $\mathfrak{B}=\overline{\phi(\mathfrak{U})}$ and $\mathfrak{U}$ is commutative, then $S(\varphi, \mathfrak{B})$ is contained in the Jacobson radical of $\mathfrak{B}$. If for each $b \in \mathfrak{B}$ we define

$$
\Delta(b)=\inf _{x \in \mathfrak{u}}(\|x\|+\|b-\nu(x)\|)
$$

then by [2], p. 1102, we must have the spectral radius of $b \leqq \Delta(b)$ for all $b \in \mathfrak{B}$. In [2] it is shown that $S(\varphi, \mathfrak{B})=\{b \in \mathfrak{B} \mid \Delta(b)=0\}$, and since $\mathfrak{B}$ is commutative, it is clear that $S(\varphi, \mathfrak{B})$ must be contained in 
the Jacobson radical of $\mathfrak{B}$. If $\mathfrak{B}$ is $C(X)$ for some compact Hausdorff $X$, then equality holds, as seen by the following proposition.

Proposition II. 1. $S(\nu, \mathfrak{B})=R$.

Proof. We need merely show that $R \subseteq S(\nu, \mathfrak{B})$. Let $r \in R$. By [1], (Th. $4.3 \mathrm{~b}$ ), there is a sequence $\left\{x_{n} \in C(X) \mid n=1,2, \cdots\right\}$ such that $\lim _{n \rightarrow \infty} \lambda\left(x_{n}\right)=r$. Letting $R(F)$ denote the dense subalgebra of $C(X)$ consisting of functions constant in some neighborhood of each point of $F$, by construction $\lambda(R(F))=0$. Since $R(F)$ is dense, choose $y_{n} \in R(F)$ such that $\lim _{n \rightarrow \infty}\left\|x_{n}-y_{n}\right\|=0$. Then

$$
\lim _{n \rightarrow \infty} \nu\left(x_{n}-y_{n}\right)=\lim _{n \rightarrow \infty} \mu\left(x_{n}-y_{n}\right)+\lim _{n \rightarrow \infty} \lambda\left(x_{n}-y_{n}\right)=\lim _{n \rightarrow \infty} \lambda\left(x_{n}\right)=r \text {, }
$$

and so $r \in S(\nu, \mathfrak{B})$.

Since $\mu$ and $\nu$ agree on a dense subalgebra, it is reasonable to suspect that their kernels are closely related. We have the following proposition.

\section{Proposition II.2. $\operatorname{Ker}(\mu)=\overline{\operatorname{Ker}(\nu)}$.}

Proof. If $\mu(x)=0$, then $\nu(x)=\lambda(x) \in R$, and if $\nu(x) \in R$, then $\mu(x)=\nu(x)-\lambda(x) \in R$, and so $\mu(x)=0$ by [1], Theorem 4.3 a. But by Proposition II.1, $R=S(\nu, \mathfrak{B})$, and so $\mu(x)=0$ if and only if $\nu(x) \in S(\nu, \mathfrak{B})$, that is, if and only if $x \in S(\nu, \mathfrak{u})$. By Theorem I.1, however, $S(\nu, \mathfrak{u})=\overline{\operatorname{Ker}(\nu)}$.

A Banach algebra homormorphism $\nu: C(X) \rightarrow \mathfrak{B}$ determines two sets that are of interest-the Badé-Curtis finite singularity set $F$, and the closed set $X_{0}$ that determines closure of the kernel of $\nu$, in the sense of Lemma I.1. We define $T(F)$ to be the algebra of all functions vanishing on some neighborhood of $F$, the neighborhood varying with the function.

Proposition II.3. $\overline{\operatorname{Ker}(\nu)} \cap T(F)=\operatorname{Ker}(\nu) \cap T(F)$.

Proof. If $x \in \overline{\operatorname{Ker}(\nu)} \cap T(F)$, by Proposition II.2,

$$
x \in \operatorname{Ker}(\mu) \cap T(F) \leqq \operatorname{Ker}(\mu) \cap R(F) .
$$

Since $\lambda$ is zero on $R(F)$, we have $\mu(x)=\lambda(x)=0$, and consequently $\nu(x)=0$.

We are now naturally led to inquire whether the singularity set 
$F$ is a subset of $X_{0}$. This is indeed the case.

Proposition II.4. $F \subseteq X_{0}$.

Proof. Let $F_{1}=F \cap X_{0}$, and let $F_{2}=F \sim F_{1}$. In order to show that $F_{2}=\varnothing$, it suffices to show that $\nu$ is continuous on $R\left(F_{1}\right)$. Since $F_{2} \cap X_{0}=\varnothing$, there exist open sets $N_{1}$ and $N_{2}$ with disjoint closures such that $F_{2} \subseteq N_{1}, X_{0} \subseteq N_{2}$. Let $f \in R\left(F_{1}\right)$ be arbitrary, and choose $g \in C(X)$ such that $g\left(\bar{N}_{1}\right)=1, g\left(\bar{N}_{2}\right)=0$. Since $g\left(N_{2}\right)=0$, by Lemma I.2 $g \in \operatorname{Ker}(\nu)$. Let $h=f-g f$. Now $h\left(N_{1}\right)=f\left(N_{1}\right)-g\left(N_{1}\right) f\left(N_{1}\right)=$ $f\left(N_{1}\right)-f\left(N_{1}\right)=0$, and since $f \in R\left(F_{1}\right)$ we see that $h \in R(F)$. Since $\nu$ is continuous on $R(F)$, there is a constant $M$ such that

$$
t \in R(F) \Rightarrow\|\nu(t)\| \leqq M\|t\|,
$$

and so $\|\nu(h)\| \leqq M\|h\|$. Since $\nu(g)=0, \nu(h)=\nu(f)-\nu(g) \nu(f)=\nu(f)$, and we also have $\|h\| \leqq(1+\|g\|)\|f\|$. Therefore

$$
\|\nu(f)\| \leqq M(1+\|g\|)\|f\|
$$

for all $f \in R\left(F_{1}\right)$, and so $F_{2}=\varnothing$.

One of the most immediate consequences of the continuity of a given homomorphism is that its kernel is closed. P. Curtis has observed to the author that, if every kernel of a homomorphism of $C(X)$ is closed, then every homomorphism of $C(X)$ is continuous. If every such kernel were closed, so would every kernel of a homomorphism of $C_{0}(Y)$ be closed, $Y$ locally compact Hausdorff. By [1], Theorem $4.3 \mathrm{c}, \lambda \mid M(F)$ is a homomorphism; closure of its kernel (which we know contains $R(F)$ ) would therefore contain $M(F)$, and so $\lambda(M(F))=0$. Given $f \in C(X)$, let $F=\left\{\omega_{i} \mid 1 \leqq i \leqq n\right\}$ be the singularity set of $\nu$. Choose $\left\{e_{i} \in C(X) \mid 1 \leqq i \leqq n\right\} \quad$ such that $i \neq j \Rightarrow e_{i} e_{j}=0,0 \leqq e_{i} \leqq 1$, and $e_{i}(\omega) \equiv 1$ in a neighborhood of $\omega_{i} \in F$. Then $f-\sum_{i=1}^{n} f\left(\omega_{i}\right) e_{i} \in M(F)$. Since $\mu$ is continuous on $C(X)$, there is a constant $M$ such that $g \in C(X) \Rightarrow\|\mu(g)\| \leqq M\|g\|$. Since

$$
f=\sum_{i=1}^{n} f\left(\omega_{i}\right) e_{i}+\left(f-\sum_{i=1}^{n} f\left(\omega_{i}\right) e_{i}\right),
$$

we have $\nu(f)=\sum_{i=1}^{n} f\left(\omega_{i}\right) \nu\left(e_{i}\right)+\mu\left(f-\sum_{i=1}^{n} f\left(\omega_{i}\right) e_{i}\right)$ and so

$$
\begin{aligned}
\| \nu((f) & \leqq\|f\| \sum_{i=1}^{n}\left\|\nu\left(e_{i}\right)\right\|+M\left\|f-\sum_{i=1}^{n} f\left(\omega_{i}\right) e_{i}\right\| \\
& \leqq\left[\sum_{i=1}^{n}\left\|\nu\left(e_{i}\right)\right\|+M(n+1)\right]\|f\|
\end{aligned}
$$

thus demonstrating the continuity of $\nu$. 
The author would like to thank the referee for suggestions and simplifications of the original proofs.

\section{REFERENCES}

1. W. G. Badé and P. C. Curtis, Jr., Homomorphisms of commutative Banach algebras, Amer. J. Math. 82 (1960), 589-608.

2. S. B. Cleveland, Homomorphisms of noncommutative *-algebras, Pacific J. Math. 13 (1963), 1097-1109.

3. L. Gillman and M. Jerison, Rings of Continuous Functions Princeton, 1962.

4. C. E. Rickart, Banach Algebras, New York, 1960.

Received September 21, 1967. The results in this paper are from the author's dissertation. The author gratefully acknowledges the support of the National Science Foundation under Grant No. GP-5138, and the direction of Professor William G. Badé of the University of California, Berkeley.

UNiversity of CALIFornia, Los ANgeles 


\section{PACIFIC JOURNAL OF MATHEMATICS}

\section{EDITORS}

\section{H. ROYDEN}

Stanford University

Stanford, California

R. R. Phelps

University of Washington

Seattle, Washington 98105

\section{J. DugundJI}

Department of Mathematics

University of Southern California

Los Angeles, California 90007

RICHARD ARENS

University of California

Los Angeles, California 90024

\section{ASSOCIATE EDITORS}
E. F. BeCKENBACH
B. H. NeumanN
F. WOLF
K. YosIDA

\section{SUPPORTING INSTITUTIONS}

\author{
UNIVERSITY OF BRITISH COLUMBIA \\ CALIFORNIA INSTITUTE OF TECHNOLOGY \\ UNIVERSITY OF CALIFORNIA \\ MONTANA STATE UNIVERSITY \\ UNIVERSITY OF NEVADA \\ NEW MEXICO STATE UNIVERSITY \\ OREGON STATE UNIVERSITY \\ UNIVERSITY OF OREGON \\ OSAKA UNIVERSITY \\ UNIVERSITY OF SOUTHERN CALIFORNIA
}

\author{
STANFORD UNIVERSITY \\ UNIVERSITY OF TOKYO \\ UNIVERSITY OF UTAH \\ WASHINGTON STATE UNIVERSITY \\ UNIVERSITY OF WASHINGTON \\ AMERICAN MATHEMATICAL SOCIETY \\ CHEVRON RESEARCH CORPORATION \\ TRW SYSTEMS \\ NAVAL WEAPONS CENTER
}

Mathematical papers intended for publication in the Pacific Journal of Mathematics should be in typed form or offset-reproduced, double spaced with large margins. Underline Greek letters in red, German in green, and script in blue. The first paragraph or two must be capable of being used separately as a synopsis of the entire paper. It should not contain references to the bibliography. Manuscripts, in duplicate if possible, may be sent to any one of the four editors. All other communications to the editors should be addressed to the managing editor, Richard Arens, University of California, Los Angeles, California 90024.

Each author of each article receives 50 reprints free of charge; additional copies may be obtained at cost in multiples of 50 .

The Pacific Journal of Mathematics is published monthly. Effective with Volume 16 the price per volume (3 numbers) is $\$ 8.00$; single issues, $\$ 3.00$. Special price for current issues to individual faculty members of supporting institutions and to individual members of the American Mathematical Society: $\$ 4.00$ per volume; single issues $\$ 1.50$. Back numbers are available.

Subscriptions, orders for back numbers, and changes of address should be sent to Pacific Journal of Mathematics, 103 Highland Boulevard, Berkeley 8, California.

Printed at Kokusai Bunken Insatsusha (International Academic Printing Co., Ltd.), 7-17, Fujimi 2-chome, Chiyoda-ku, Tokyo, Japan.

PUBLISHED BY PACIFIC JOURNAL OF MATHEMATICS, A NON-PROFIT CORPORATION

The Supporting Institutions listed above contribute to the cost of publication of this Journal, but they are not owners of publishers and have no responsibility for its content or policies. 


\section{Pacific Journal of Mathematics}

\section{Vol. 26, No. $3 \quad$ BadMonth, 1968}

Leonard Asimow, Universally well-capped cones ................. 421

Lawrence Peter Belluce, William A. Kirk and Eugene Francis Steiner,

Normal structure in Banach spaces ..................... 433

William Jay Davis, Bases in Hilbert space.................... 441

Larry Lee Dornhoff, p-automorphic p-groups and homogeneous

algebras..................................... 447

William Grady Dotson, Jr. and W. R. Mann, A generalized corollary of the

Browder-Kirk fixed point theorem ....................... 455

John Brady Garnett, On a theorem of Mergelyan ................. 461

Matthew Gould, Multiplicity type and subalgebra structure in universal

algebras............................................. 469

Marvin D. Green, A locally convex topology on a preordered space . . . . . 487

Pierre A. Grillet and Mario Petrich, Ideal extensions of semigroups . . . . . . 493

Kyong Taik Hahn, A remark on integral functions of several complex

variables ... . . . . . . . . . . . . . . . . . . . . . . . . . . . . . . . . . . . 509

Choo Whan Kim, Uniform approximation of doubly stochastic

operators..................................... 515

Charles Alan McCarthy and L. Tzafriri, Projections in $\mathscr{L}_{1}$ and

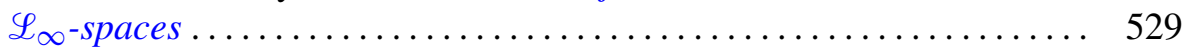

Alfred Berry Manaster, Full co-ordinals of RETs ................ 547

Donald Steven Passman, $p$-solvable doubly transitive permutation

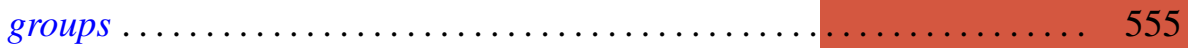

Neal Jules Rothman, An $L^{1}$ algebra for linearly quasi-ordered compact

semigroups ....................................... 579

James DeWitt Stein, Homomorphisms of semi-simple algebras .......... 589

Jacques Tits and Lucien Waelbroeck, The integration of a Lie algebra

representation ...............................

David Vere-Jones, Ergodic properties of nonnegative matrices. II ........ 601

Donald Rayl Wilken, The support of representing measures for $R(X) \ldots \ldots 621$

Abraham Zaks, Simple modules and hereditary rings .... . . 